CORRECTION

https://doi.org/10.1038/s41586-018-0402-x

\title{
Author Correction: Direct \\ conversion of human fibroblasts to multilineage blood progenitors
}

Eva Szabo, Shravanti Rampalli, Ruth M. Risueño,

Angelique Schnerch, Ryan Mitchell, Aline Fiebig-Comyn,

Marilyne Levadoux-Martin \& Mickie Bhatia

Correction to: Nature https://doi.org/10.1038/nature09591,

published online 07 November 2010.

It has been pointed out that in Supplementary Fig. 3b of this Article, some gel images of individual PCR products were inaccurately compiled, leading to duplication of gel images in lanes where no band was present. Unfortunately, the files containing the original PCR images for Supplementary Figs. $2 \mathrm{e}$ and $3 \mathrm{~b}$ could not be located. We have therefore repeated these experiments using the same human cellular reagents as originally reported to confirm our results and conclusions. We provide the raw images of gel products, as well as the flow cytometry, human embryoid body images, and flowchart of the replication of these experiments, in the Supplementary Information of this Amendment. We thank M. Nakanishi and T. Collins for independently performing these experiments and analysing the results. The original Article has not been corrected.

Supplementary information is available for this Amendment at https://doi.org/ $10.1038 / \mathrm{s} 41586-018-0402-x$ 